\title{
Constructing quantum games from symmetric non-factorizable joint probabilities
}

\author{
James M. Chappell* \\ School of Chemistry and Physics, University of Adelaide, South Australia 5005, Australia
}

\author{
Azhar Iqbal \\ a) School of Electrical and Electronic Engineering, \\ University of Adelaide, South Australia 5005, Australia \\ b) Centre for Advanced Mathematics and Physics, \\ National University of Sciences $\& 3$ Technology, \\ Peshawar Road, Rawalpindi, Pakistan
}

Derek Abbott

School of Electrical and Electronic Engineering, University of Adelaide 5005, Australia

(Dated: October 26, 2018)

\begin{abstract}
We construct quantum games from a table of non-factorizable joint probabilities, coupled with a symmetry constraint, requiring symmetrical payoffs between the players. We give the general result for a Nash equilibrium and payoff relations for a game based on non-factorizable joint probabilities, which embeds the classical game. We study a quantum version of Prisoners' Dilemma, Stag Hunt, and the Chicken game constructed from a given table of non-factorizable joint probabilities to find new outcomes in these games. We show that this approach provides a general framework for both classical and quantum games without recourse to the formalism of quantum mechanics.
\end{abstract}

PACS numbers: 03.67.Lx

*Electronic address: james.m.chappell@adelaide.edu.au 


\section{INTRODUCTION}

As an established branch of mathematics, game theory [1, 2] analyzes strategic decision making of competing agents who find themselves in conflict situations. Game theory finds extensive applications in social sciences, biology and engineering. Recent developments in quantum computing and quantum information theory [3] have motivated efforts to recast classical game theory using quantum probability amplitudes [4], leading to the birth of the area of quantum games [5-37] . Quantum games have been proposed [7] as a new way to approach quantum algorithms, including speculation that nature may be playing quantum games at the molecular level [38].

In the area of quantum games, a recently reported [31] probabilistic approach constructs them from a set of non-factorizable joint probabilities. It is a set that cannot be factorized in terms of other more fundamental probabilities. The motivating idea being that Bell's inequalities can be violated by a set of non-factorizable probabilities, though this does not imply that any non-factorizable set will violate the inequalities. As the violation of Bell's inequalities is considered a fundamentally quantum aspect, one is motivated to have an approach to quantum games that constructs them from the property of a probability set of being non-factorizable. This then provides greater mathematical generality for quantum games, providing accessibility without the formalism of quantum mechanics.

The earliest quantization attempts [8] were focussed at the two-player two-strategy $(2 \times 2)$ non-cooperative games . The playing of a $2 \times 2$ game can be easily realized if players share a physical system that involves 4 joint probabilities. This, for instance, will be the case when players share two coins that can be put in head $(\mathcal{H})$ and tail $(\mathcal{T})$ states. A referee gives each player a coin and asks them to flip it to either the $\mathcal{H}$ or the $\mathcal{T}$ state. As no tossing but only flipping actions are involved, it does not matter if the coins are biased or not. After a players' flipping (or not-flipping) actions the coins can be found in a $\mathcal{H} \mathcal{H}, \mathcal{H} \mathcal{T}, \mathcal{T H}$, or $\mathcal{T} \mathcal{T}$ state, where the first entry in each pair, is reserved for the state of Alice's coin. From a given $2 \times 2$ game table, the referee can then award players their payoffs depending on the state of the two coins.

The probabilistic approach to quantum games developed in Ref. [31] extends the playing of a $2 \times 2$ game towards the quantum domain by considering two players who share a bigger physical system that also involves coin tossing. Consider four biased coins that two players 
share to play a $2 \times 2$ game according to the following arrangement. In a run, each player is given two coins and she/he has to select one. The referee tosses the two selected coins together and records the outcome. It can then be shown that the players' payoffs, in a mixed-strategy version of the $2 \times 2$ game, can be expressed in terms of their strategic choices performed over multiple runs and the relevant 16 factorizable joint probabilities.

In order to maintain the bilinear payoff structure of the $2 \times 2$ game, constraints are placed on joint probabilities. Allowing joint probabilities to become non-factorizable, while they remain under these constraints, ensures that the classical payoffs and the outcome of the game are obtained when the probabilities become factorizable.

In the present paper, we introduce an extra condition requiring that the considered joint probabilities are also symmetric both when they are factorizable and non-factorizable. We argue that this is a natural constraint to be included in a probabilistic approach to quantum games that builds them from non-factorizable joint probabilities. We find that this constraint further narrows down our focus in obtaining a quantum game from probabilistic considerations only. We study quantum versions of the Prisoners' Dilemma (PD) game, the Stag Hunt (SH) game, and the Chicken game [1, 2] within this approach that constructs them from not only non-factorizable but also symmetric joint probabilities. We investigate how non-factorizable and symmetric joint probabilities permit new equilibria in these games. By introducing parameters that present a measure of non-factorizability, we discuss a novel way of obtaining a set of non-factorizable joint probabilities, which allows us to identify factorizable, non-factorizable, and the quantum domains.

\section{AN APPROACH TOWARDS EXTENDING A $2 \times 2$ GAME}

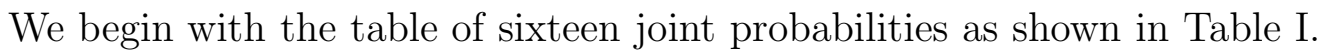

For Alice and Bob we also have payoff matrices given by

$$
\begin{aligned}
& \text { Bob Bob } \\
& S_{1}^{\prime} S_{2}^{\prime} \quad S_{1}^{\prime} S_{2}^{\prime} \\
& \mathcal{A}=\text { Alice } \begin{array}{l}
S_{1} \\
S_{2}
\end{array}\left(\begin{array}{ll}
a_{1} & a_{2} \\
a_{3} & a_{4}
\end{array}\right), \quad \mathcal{B}=\text { Alice } \begin{array}{l}
S_{1} \\
S_{2}
\end{array}\left(\begin{array}{ll}
b_{1} & b_{2} \\
b_{3} & b_{4}
\end{array}\right),
\end{aligned}
$$

giving Alice's and Bob's payoffs, respectively. Because we are considering games with symmetrical payoffs we have $\mathcal{B}=\mathcal{A}^{T}$, where $T$ indicates transpose. This requires 


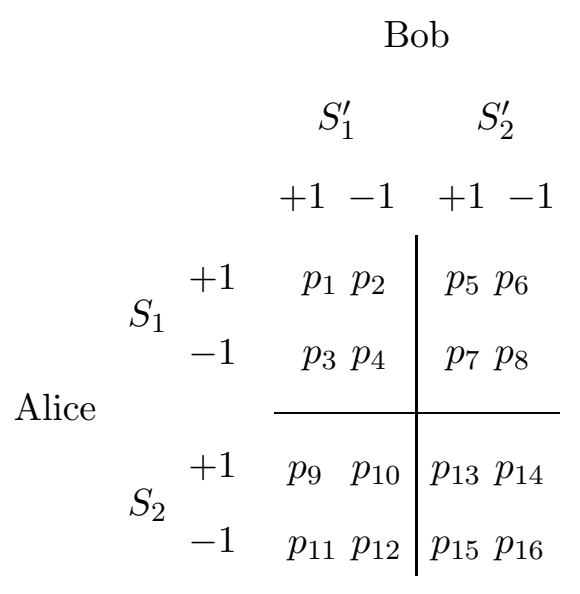

TABLE I: General probability table.

$$
b_{1}=a_{1}, b_{2}=a_{3}, b_{3}=a_{2}, b_{4}=a_{4}
$$

In a mixed-strategy game one has the strategy vectors $\mathbf{x}=(x, 1-x)^{T}$ and $\mathbf{y}=(y, 1-y)^{T}$, with $x, y \in[0,1]$ giving the probabilities for Alice and Bob to choose $S_{1}$ and $S_{1}^{\prime}$ respectively. Payoff relations in a mixed-strategy game are

$$
\Pi_{A, B}(x, y)=\mathbf{x}^{T}(\mathcal{A}, \mathcal{B}) \mathbf{y}
$$

where subscripts $A$ and $B$ refer to Alice and Bob, respectively. In this notation we can, for instance, have the pure strategy payoffs $\Pi_{A}\left(S_{1}, S_{1}^{\prime}\right)=\Pi_{A}(1,1)=a_{1}=\Pi_{B}(1,1)$ etc. We represent strategies $\mathbf{x}$ and $\mathbf{y}$ by numbers $x$ and $y$ respectively and note that the strategy pair $\left(x^{\star}, y^{\star}\right)$ is a Nash equilibrium $(\mathrm{NE})$ when

$$
\Pi_{A}\left(x^{\star}, y^{\star}\right)-\Pi_{A}\left(x, y^{\star}\right) \geq 0, \quad \Pi_{B}\left(x^{\star}, y^{\star}\right)-\Pi_{B}\left(x^{\star}, y\right) \geq 0 .
$$

A possible physical realization for playing this symmetric game uses two coins in the following arrangement. The referee announces the association $S_{1}, S_{1}^{\prime} \sim \mathcal{H}$ and $S_{2}, S_{2}^{\prime} \sim \mathcal{T}$ and each player's strategy consists of secretly flipping his/her penny either to the $\mathcal{H}$ or to the $\mathcal{T}$ state. The players then simultaneously return their pennies to the referee. The referee observes the state of the two coins and rewards the players. In the case of pure strategies the referee can use the matrices (2) and in case of mixed strategies, the players are rewarded according to the payoff relations (44). 
As mentioned in the introduction, the referee can also have a different arrangement that allows the playing the game using four coins instead of two, as follows. S/he identifies the four coins as $S_{1}, S_{2} ; S_{1}^{\prime}, S_{2}^{\prime}$ (note that $S_{1}$ and $S_{2}$ are no longer a player's actions to put his/her penny in $\mathcal{H}$ or $\mathcal{T}$ state). In a run s/he gives coins $S_{1}, S_{2}$ to Alice and coins $S_{1}^{\prime}, S_{2}^{\prime}$ to Bob. Each player now has to choose one out of the two coins so that the chosen pair is one of the $\left(S_{1}, S_{1}^{\prime}\right),\left(S_{1}, S_{2}^{\prime}\right),\left(S_{2}, S_{1}^{\prime}\right),\left(S_{2}, S_{2}^{\prime}\right)$. The players return the two chosen coins to the referee who tosses them together and records the outcome. The referee then collects four coins (two tossed and two untossed) and prepares them for the next run. In this extended game, players' payoff relations can now be defined by making the association $\mathcal{H} \sim+1 \& \mathcal{T} \sim-1$ and using the 16 joint probabilities $p_{1}, p_{2}, \ldots p_{16}$, as described in Table (II).

We write the payoffs relations as,

$$
\begin{array}{ll}
\Pi_{A, B}\left(S_{1}, S_{1}^{\prime}\right)=\sum_{i=1}^{4}(a, b)_{i} p_{i}, & \Pi_{A, B}\left(S_{1}, S_{2}^{\prime}\right)=\sum_{i=5}^{8}(a, b)_{i-4} p_{i}, \\
\Pi_{A, B}\left(S_{2}, S_{1}^{\prime}\right)=\sum_{i=9}^{12}(a, b)_{i-8} p_{i}, & \Pi_{A, B}\left(S_{2}, S_{2}^{\prime}\right)=\sum_{i=13}^{16}(a, b)_{i-12} p_{i},
\end{array}
$$

where $\Pi_{B}\left(S_{2}, S_{1}^{\prime}\right)$, for instance, corresponds when Alice selects her $S_{2}$ coin and Bob selects his $S_{1}^{\prime}$ coin over all the runs. In Eqs. (6) each of the four payoff relations give mixedstrategy payoffs of the $2 \times 2$ game. Over many runs, the players can also select a probability distribution over the available strategies and one can define

$$
\Pi_{A, B}(x, y)=\mathbf{x}^{T}\left(\begin{array}{ll}
\Pi_{A, B}\left(S_{1}, S_{1}^{\prime}\right) & \Pi_{A, B}\left(S_{1}, S_{2}^{\prime}\right) \\
\Pi_{A, B}\left(S_{2}, S_{1}^{\prime}\right) & \Pi_{A, B}\left(S_{2}, S_{2}^{\prime}\right)
\end{array}\right) \mathbf{y}
$$

where $x \in[0,1]$ is the probability with which Alice selects coins $S_{1}$ and $y \in[0,1]$ is the probability with which Bob selects coin $S_{1}^{\prime}$. Eqs. (7) give mixed-strategy payoffs in an extension of the original $2 \times 2$ game. As discussed in Refs. [31, 33, 35], this extension involving 16 joint factorizable probabilities can be considered a re-expression of the classical game that transforms the original game in such a way that a transition to the quantum game is achievable by a consideration of non-factorizable joint probabilities. This re-expressed game with factorizable probabilities is of course classically implementable and is not to be confused with the original game from which it is derived. 


\section{A. Obtaining a symmetric game}

Enforcing game symmetry, under the interchange of players, we require $\Pi_{A}\left(S_{i}, S_{j}^{\prime}\right)=$ $\Pi_{B}\left(S_{j}, S_{i}^{\prime}\right)$ and thus we note from Eqs. (6) that this is achievable if

$$
p_{2}=p_{3} ; \quad p_{5}=p_{9}, p_{6}=p_{11}, p_{7}=p_{10}, p_{8}=p_{12} ; \quad p_{14}=p_{15}
$$

With this we produce Table (III) and notice that the table of probabilities is now symmetric across the main diagonal. By including the normalization constraint on each quadrant, we

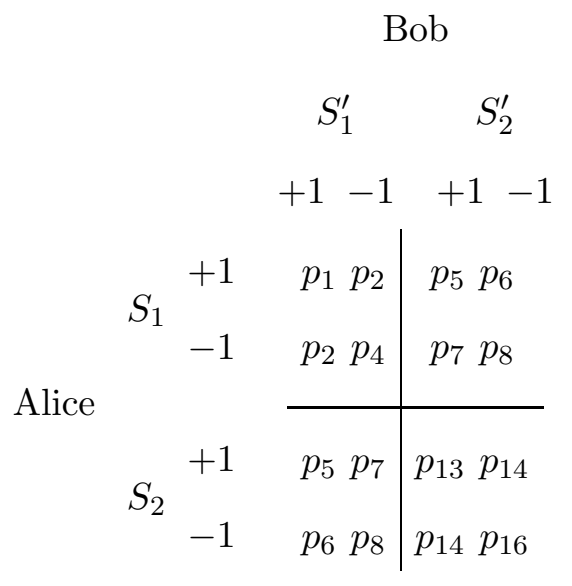

TABLE II: Symmetric probability table.

produce Table (III).

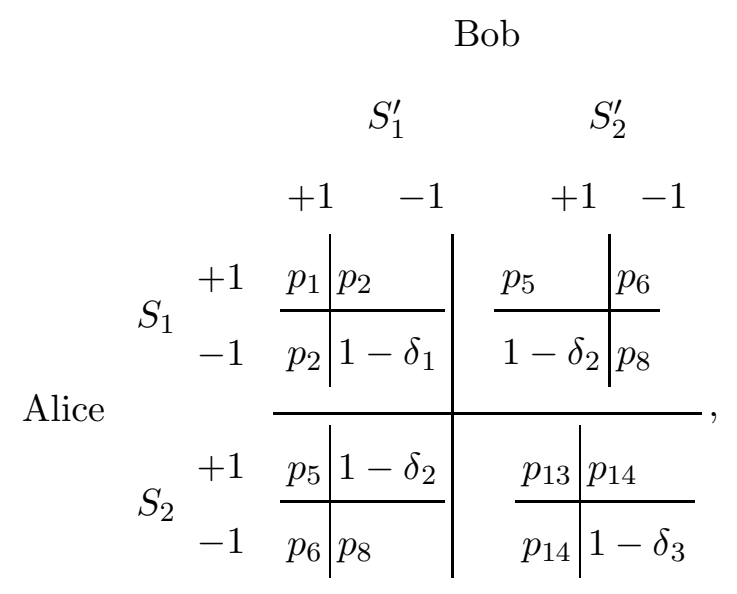

TABLE III: Symmetric normalised probability table. Here $\delta_{1}=p_{1}+2 p_{2}, \delta_{2}=p_{5}+p_{6}+p_{8}$, and $\delta_{3}=p_{13}+2 p_{14}$. 
This arrangement for playing a $2 \times 2$ game with 16 joint probabilities facilitates a transition to playing the same game using an Einstein-Podolsky-Rosen (EPR) type setup [4, 40-43]. In this setup, Alice and Bob are spatially separated and are unable to communicate with each other. In an individual run, both receive one half of a pair of particles originating from a common source. In the same run of the experiment, both choose one from two given (pure) strategies. These strategies are the two directions in space along which spin or polarization measurements can be made. Keeping the notation for the coins, we denote these directions to be $S_{1}, S_{2}$ for Alice and $S_{1}^{\prime}, S_{2}^{\prime}$ for Bob. Each measurement generates +1 or -1 as the outcome, as it is the case with coins after their toss in the four-coin setup. Experimental outcomes are recorded for a large number of individual runs and payoffs are awarded according to the directions the players choose over many runs (defining their strategies), the matrix of the game they play, and the statistics of the measurement outcomes.

When $p_{i}(1 \leq i \leq 16)$ are taken as the EPR probabilities, they of course satisfy the normalization constraint, stating that the sums $\sum_{i=1}^{4} p_{i}, \sum_{i=5}^{8} p_{i}, \sum_{i=9}^{12} p_{i}$, and $\sum_{i=13}^{16} p_{i}$ are all equal to 1. EPR probabilities also satisfy other constraints imposed by the requirements of causality, stating that Alice's outcome of +1 or -1 (obtained along $S_{1}$ or $S_{2}$ ) is independent of whether Bob chooses $S_{1}^{\prime}$ or $S_{2}^{\prime}$, and similarly Bob's outcome of +1 or -1 (obtained along $S_{1}^{\prime}$ or $S_{2}^{\prime}$ ) is independent of whether Alice chooses $S_{1}$ or $S_{2}$. This can be written as

$$
\begin{array}{ll}
p_{1}+p_{2}=p_{5}+p_{6}, & p_{1}+p_{3}=p_{9}+p_{11}, \\
p_{9}+p_{10}=p_{13}+p_{14}, & p_{5}+p_{7}=p_{13}+p_{15}, \\
p_{3}+p_{4}=p_{7}+p_{8}, & p_{11}+p_{12}=p_{15}+p_{16}, \\
p_{2}+p_{4}=p_{10}+p_{12}, & p_{6}+p_{8}=p_{14}+p_{16},
\end{array}
$$

which is also referred to as the causal communication constraint [43]. These provide two more dependencies giving $p_{6}=p_{1}+p_{2}-p_{5}$ and $p_{8}=p_{14}+p_{16}-p_{6}$.

Note that the causal communication constraints (11) are set into two groups. The first group states that the probability of obtaining a particular outcome $(+1$ or -1$)$ on Alice's side of the EPR type apparatus is independent of which one of the two measurements are performed on Bob's side. Similarly, the second group states that the probability of obtaining a particular outcome $(+1$ or -1$)$ on Bob's side of the EPR type apparatus is independent of which one of the two measurements are performed on Alice's side. These constraints hold even when Alice and Bob share an entangled state, and the non-factorizable probability sets we consider below to construct quantum games always respect the causal 
communication constraints. The causal communication constraint is also sometimes referred to as 'parameter independence', 'simple locality','signal locality' or 'physical locality' and prevents the acausal exchange of classical information between different parts of a quantum system. This fundamental constraint is therefore retained even for the cases when the probabilities become non-factorizable.

Substituting these relations we finally obtain the probability table for symmetric games given by Table (IV) in terms of the five independent variables $p_{1}, p_{2}, p_{5}, p_{13}, p_{14}$.

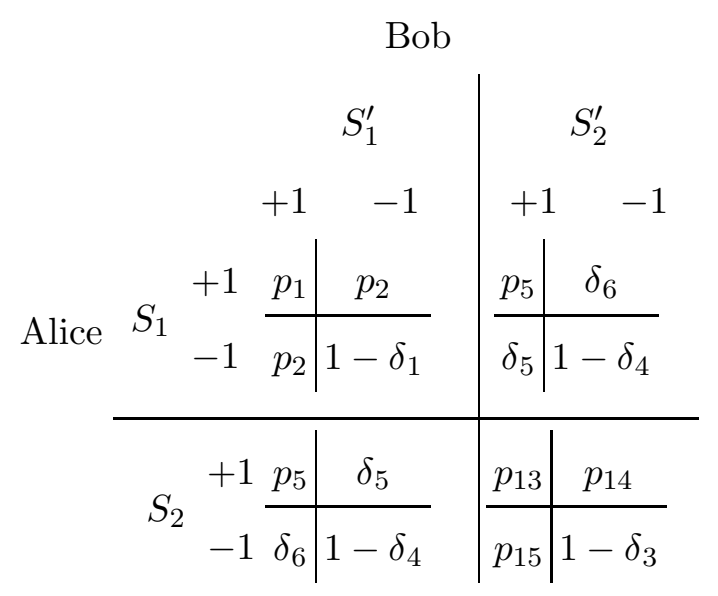

TABLE IV: Symmetric normalised causal probability table. Here $\delta_{4}=p_{1}+p_{2}-p_{5}+p_{13}+p_{14}$, $\delta_{5}=-p_{5}+p_{13}+p_{14}$, and $\delta_{6}=p_{1}+p_{2}-p_{5}$

If $x, y \in[0,1]$ are defined to be the probability to select $S_{1}$ over $S_{2}$ and $S_{1}^{\prime}$ over $S_{2}^{\prime}$ by Alice and Bob respectively, then we have Alice's expected payoff given by

$\Pi_{A}(x, y)=x y \Pi_{A}\left(S_{1}, S_{1}^{\prime}\right)+x(1-y) \Pi_{A}\left(S_{1}, S_{2}^{\prime}\right)+(1-x) y \Pi_{A}\left(S_{2}, S_{1}^{\prime}\right)+(1-x)(1-y) \Pi_{A}\left(S_{2}, S_{2}^{\prime}\right)$.

Bob's payoff is then obtained from above by interchanging $x$ and $y$. Substituting Eqs. (6) and re-arranging we find

$$
\begin{aligned}
\Pi_{A}(x, y) & =x y \Delta_{3} v_{3}+x\left(\Delta_{1} v_{1}-\Delta_{2} v_{2}\right)+y\left\{\left(a_{2}-a_{1}\right) v_{1}+\left(a_{3}-a_{4}\right) v_{2}\right\} \\
& +a_{1} p_{13}+\left(a_{2}+a_{3}\right) p_{14}+a_{4}\left(1-p_{13}-2 p_{14}\right)
\end{aligned}
$$

where $\Delta_{1}=a_{3}-a_{1}, \Delta_{2}=a_{4}-a_{2}$ and $\Delta_{3}=\Delta_{2}-\Delta_{1}$ with

$$
v_{1}=w, v_{2}=u+v, v_{3}=u+w
$$


where $u=p_{1}-p_{5}, v=p_{2}-p_{14}$ and $w=p_{13}-p_{5}$. By symmetry we also have for Bob

$$
\begin{aligned}
\Pi_{B}(x, y) & =x y \Delta_{3} v_{3}+y\left(\Delta_{1} v_{1}-\Delta_{2} v_{2}\right)+x\left\{\left(a_{2}-a_{1}\right) v_{1}+\left(a_{3}-a_{4}\right) v_{2}\right\} \\
& +a_{1} p_{13}+\left(a_{2}+a_{3}\right) p_{14}+a_{4}\left(1-p_{13}-2 p_{14}\right) .
\end{aligned}
$$

For a NE we need to satisfy the relations

$$
\begin{aligned}
& \Pi_{A}\left(x^{*}, y^{*}\right)-\Pi_{A}\left(x, y^{*}\right)=\left(x^{*}-x\right)\left[y^{*} \Delta_{3} v_{3}+\Delta_{1} v_{1}-\Delta_{2} v_{2}\right] \geq 0 \\
& \Pi_{B}\left(x^{*}, y^{*}\right)-\Pi_{B}\left(x^{*}, y\right)=\left(y^{*}-y\right)\left[x^{*} \Delta_{3} v_{3}+\Delta_{1} v_{1}-\Delta_{2} v_{2}\right] \geq 0
\end{aligned}
$$

We thus have the NE defined for symmetric games with the three variables $v_{1}, v_{2}, v_{3}$.

\section{B. When probabilities are factorizable}

If the probability table is factorizable then we can write

$$
\begin{gathered}
p_{1}=r r^{\prime}, p_{2}=r\left(1-r^{\prime}\right), p_{3}=r^{\prime}(1-r), p_{4}=(1-r)\left(1-r^{\prime}\right) \\
p_{5}=r s^{\prime}, p_{6}=r\left(1-s^{\prime}\right), p_{7}=s^{\prime}(1-r), p_{8}=(1-r)\left(1-s^{\prime}\right) \\
p_{9}=s r^{\prime}, p_{10}=s\left(1-r^{\prime}\right), p_{11}=r^{\prime}(1-s), p_{12}=(1-s)\left(1-r^{\prime}\right) \\
p_{13}=s s^{\prime}, p_{14}=s\left(1-s^{\prime}\right), p_{15}=s^{\prime}(1-s), p_{16}=(1-s)\left(1-s^{\prime}\right),
\end{gathered}
$$

where $r, s, r^{\prime}, s^{\prime} \in[0,1]$ and $r=p_{1}+p_{2}$ and $s=p_{13}+p_{14}$. From symmetry we have $p_{2}=p_{3}$ which immediately implies $r=r^{\prime}$ and also $s=s^{\prime}$ from $p_{14}=p_{15}$.

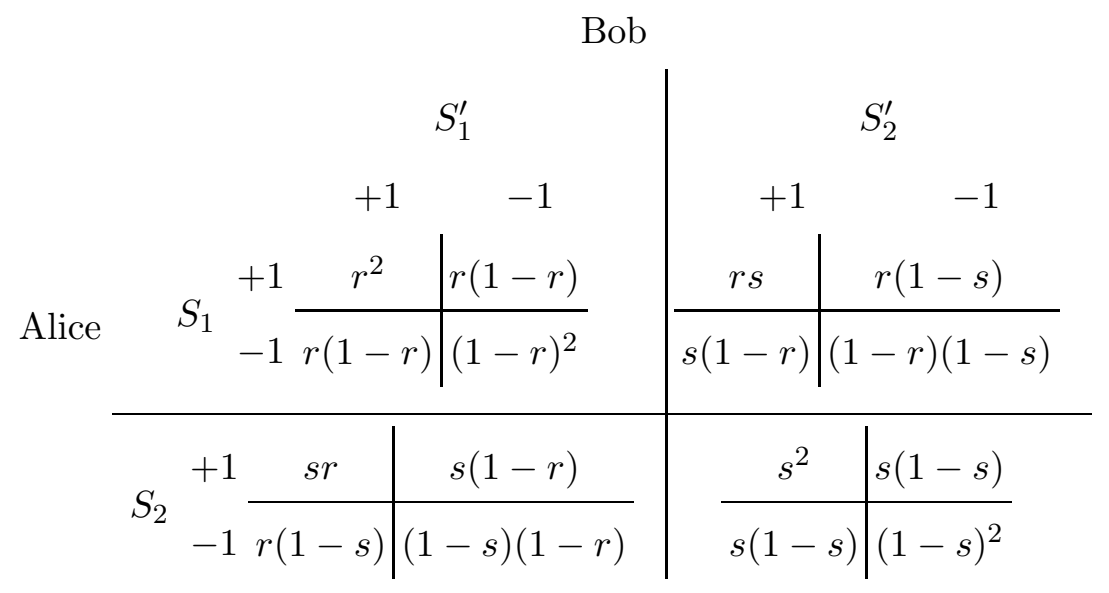

TABLE V: Factorizable probabilities. 
So we can now find from Eqs. (15) $v_{1}=-s(r-s), v_{2}=(r-s)(1-s)$ and $v_{3}=(r-s)^{2}$. Substituting these results into Eq. (17) gives the following conditions for the strategy pair $\left(x^{*}, y^{*}\right)$ to be a $\mathrm{NE}$

$$
\begin{aligned}
& \Pi_{A}\left(x^{*}, y^{*}\right)-\Pi_{A}\left(x, y^{*}\right)=\left(x^{*}-x\right)(r-s)\left[\Delta_{3}\left\{y^{*} r+\left(1-y^{*}\right) s\right\}-\Delta_{2}\right] \geq 0, \\
& \Pi_{B}\left(x^{\star}, y^{\star}\right)-\Pi_{B}\left(x^{\star}, y\right)=\left(y^{*}-y\right)(r-s)\left[\Delta_{3}\left\{x^{*} r+\left(1-x^{*}\right) s\right\}-\Delta_{2}\right] \geq 0 .
\end{aligned}
$$

These are the defining equations for a NE when assuming symmetry and factorizability.

For Alice, we have the payoff in the factorizable case

$$
\begin{aligned}
\Pi_{A}(x, y) & \left.=x y \Delta_{3}(r-s)^{2}+x(r-s)\left(\Delta_{3} s-\Delta_{2}\right)+y(r-s)\left(\Delta_{3} s+a_{3}-a_{4}\right)\right] \\
& +a_{4}-s\left(\Delta_{2}-a_{3}+a_{4}\right)+\Delta_{3} s^{2}
\end{aligned}
$$

and a similar expression for Bob is obtained by exchanging $x$ for $y$.

\section{Obtaining the classical mixed strategy game}

To achieve the classical payoff structure we see from the first term in Eq. (22) that $(r-s)^{2}=1$, which requires $r=1$ and $s=0$ to give the payoff

$$
\Pi_{A}(x, y)=a_{4}+x\left(a_{2}-a_{4}\right)+y\left(a_{3}-a_{4}\right)+x y\left(a_{1}-a_{2}-a_{3}+a_{4}\right),
$$

giving the required classical bilinear payoff structure, which has associated NE given by

$$
\left(x^{*}-x\right)\left[\Delta_{3} y^{*}-\Delta_{2}\right] \geq 0 .
$$

\section{Prisoners' Dilemma}

For the PD game we have $\Delta_{1}, \Delta_{2}>0$ and hence $\left|\Delta_{3}\right| \leq \Delta_{2}$. This makes the term in the square bracket in Eq. (20) and Eq. (21) to be always negative, hence we just require $r>s$ if $\left(x^{*}, y^{*}\right)=(0,0)$ is to exist as a NE. The condition $r>s$ implies that the coins are basically in a heads up state, which is obviously reasonable because if we invert the coins before the game then we invert the NE. This shows that symmetry and factorizability along with the condition $r>s$ will return the classical NE for the PD game. 


\section{Stag Hunt}

For the SH game we have $\Delta_{3}>\Delta_{2}>0$ and $\Delta_{1}+\Delta_{2}>0$ and $\Delta_{3}>\Delta_{1}+\Delta_{2}$. For mixed NE we require from Eq. (201) $y^{*} \Delta_{3}(r-s)+\Delta_{3} s-\Delta_{2}=0$ or

$$
y^{*}=\left(\Delta_{2} / \Delta_{3}-s\right) /(r-s)
$$

Because, by definition, $y^{*} \geq 0$ then this requires $s \leq \Delta_{2} / \Delta_{3}$ and similarly because $y^{*} \leq 1$ then $\left[\Delta_{2}-s \Delta_{3}\right] /\left[\Delta_{3}(r-s)\right] \leq 1$ or $\Delta_{2} \leq \Delta_{3} r$ or

$$
r \geq \Delta_{2} / \Delta_{3}
$$

To create the classical mixed NE in the classical game, we define $r=\Delta_{2} / \Delta_{3}+\left(1-\Delta_{2} / \Delta_{3}\right) g$ where $g \in(0,1]$ and $s=\Delta_{2} / \Delta_{3}(1-h)$ where $h \in(0,1]$. This gives us $\left(\Delta_{2} / \Delta_{3}-s\right) /(r-s)=$ $\Delta_{2} / \Delta_{3}$ or $h \Delta_{2} / \Delta_{3} /\left(\left(1-\Delta_{2} / \Delta_{3}\right) g+\Delta_{2} / \Delta_{3} h\right)=\Delta_{2} / \Delta_{3}$ or that $g=h$. This result indicates that $r$ and $s$ are a proportional distance from $\Delta_{2} / \Delta_{3}$. This then gives that

$$
s=\frac{1-r}{\Delta_{3} / \Delta_{2}-1} .
$$

For the other NE, $\left(x^{*}, y^{*}\right)=(0,0)$, if we have $y^{*}=0$ then we require from Eq. (20)

$$
\Pi_{A}\left(x^{*}, y^{*}\right)-\Pi_{A}\left(x, y^{*}\right)=\frac{1}{2}\left(x^{*}-x\right)(r-s)\left[\Delta_{3} s-\Delta_{2}\right] \geq 0,
$$

and in order to return $x^{*}=0$ requires $s<\Delta_{2} / \Delta_{3}$. Also, if we have $y^{*}=1$ then we require

$$
\Pi_{A}\left(x^{*}, y^{*}\right)-\Pi_{A}\left(x, y^{*}\right)=\frac{1}{2}\left(x^{*}-x\right)(r-s)\left[\Delta_{3} r-\Delta_{2}\right] \geq 0,
$$

and in order to return $x^{*}=1$ requires $r>\Delta_{2} / \Delta_{3}$. Hence we find three NE

$$
\begin{aligned}
& \left(x^{*}, y^{*}\right)=(0,0) \\
& \left(x^{*}, y^{*}\right)=\left(\Delta_{2} / \Delta_{3}, \Delta_{2} / \Delta_{3}\right) \\
& \left(x^{*}, y^{*}\right)=(1,1)
\end{aligned}
$$

conditional on Eq. (26) and Eq. (27). We know $0<\Delta_{2} / \Delta_{3}<1$, hence we can always find an $r$ and an $s$ to create this particular classical game. 


\section{Chicken game}

For the Chicken game we have $\Delta_{3}=-(\alpha+\beta)<0$ and $\Delta_{2}=-\alpha<0$ and $\Delta_{1}=\beta>0$, where $\alpha, \beta>0$. The general condition for NE is obtained from Eq. (20) as

$$
\frac{1}{2}\left(x^{*}-x\right)(r-s)\left[-y^{*}(\alpha+\beta)(r-s)-(\alpha+\beta) s+\alpha\right] \geq 0 .
$$

We can see that $\Delta_{2} / \Delta_{3}=\alpha /(\alpha+\beta)$ hence we will duplicate the results of the previous $\mathrm{SH}$ game, obtaining the correct classical NE

$$
\begin{aligned}
& \left(x^{*}, y^{*}\right)=(1,0) \\
& \left(x^{*}, y^{*}\right)=(\alpha /(\alpha+\beta), \alpha /(\alpha+\beta)) \\
& \left(x^{*}, y^{*}\right)=(0,1)
\end{aligned}
$$

provided $r>\alpha /(\alpha+\beta)$ and $s=(1-r) /\{(\alpha+\beta) / \alpha-1\}$.

\section{Discussion}

We find $\alpha /(\alpha+\beta)=\Delta_{2} / \Delta_{3}=\left(a_{4}-a_{2}\right) /\left(a_{4}-a_{2}-a_{3}+a_{1}\right)$, hence for the three games studied, if we select $r$ such that $\left(a_{4}-a_{2}\right) /\left(a_{4}-a_{2}-a_{3}+a_{1}\right)<r \leq 1$ with $s$ given by Eq. (27), in each case we will return the classical NE for these three games when the probability table becomes factorizable, although not the classical bilinear payoffs. If we also require this payoff structure, then we require the more restrictive constraint $r=1$ and $s=0$.

\section{EXTENSION TOWARDS NON-FACTORIZABLE JOINT PROBABILITIES}

We have shown that factorizability along with symmetry and the conditions $r=1$ and $s=0$ embeds the classical game within the quantum game. If we enforce $r=p_{1}+p_{2}=1$ in the general quantum game, then we have $p_{3}=p_{4}=0$ by normalization, but by symmetry $p_{2}=0$, and therefore $p_{1}=1$, similarly for the rest of the table. Hence this condition creates the table of factorizable probabilities with $p_{1}=p_{6}=p_{11}=p_{16}=1$ and all other probabilities zero.

However we can still create a non-factorizable set of probabilities by inserting offset parameters from the starting position in the Table (V]). We now add extra parameters into 
Table $\mathrm{V}$ exploiting any available degrees of freedom. For the upper left quadrant because we are constrained by normalization, symmetry, and the causal communication constraint, we only have available two degrees of freedom. This is utilized with the parameters $a$ and $b$ as shown in Table ( $(\mathrm{VI})$. We then continue this process and we find that we can add up to 5 independent parameters, $a, b, c, d, e \in \Re$ in the range $[-1,1]$.

Bob

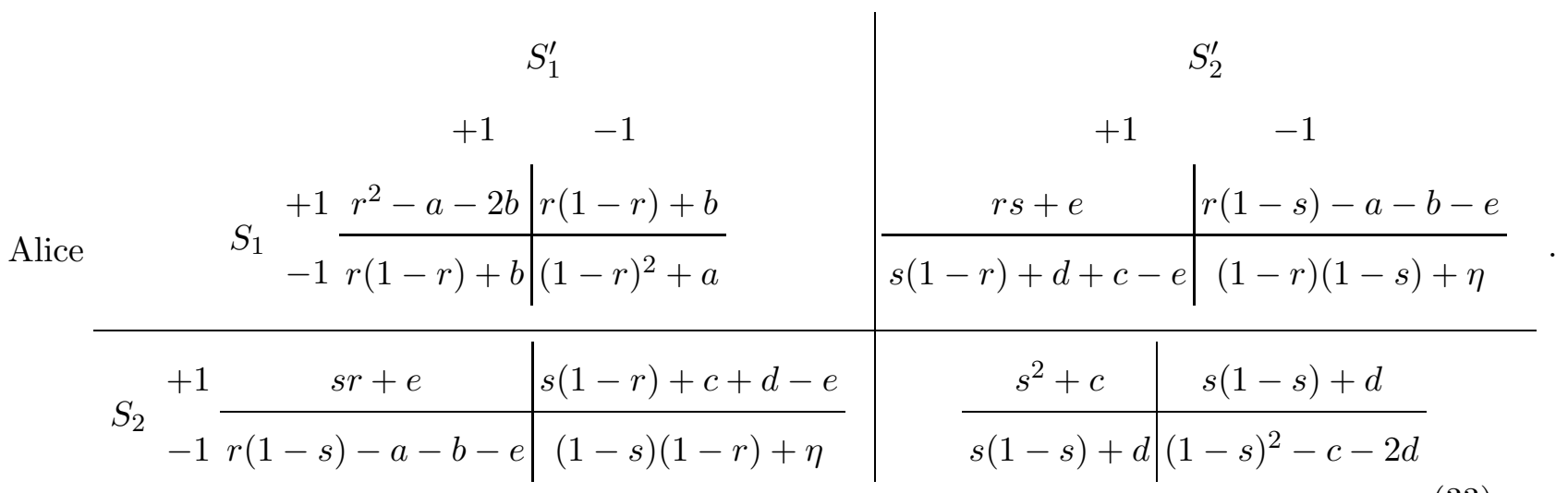

TABLE VI: Parameterizing non-factorizability.

In Table (VI) $\eta=a+b+e-c-d$, and $a, b, c, d, e$ chosen such that each one of the 16 probabilities in Table ( $(\overline{\mathrm{VI}})$ remains in the range $[0,1]$.

From Table (VI) and Eqs. (15) we find

$$
v_{1}=-s(r-s)-\epsilon_{1}, v_{2}=(1-s)(r-s)-\epsilon_{2}, v_{3}=(r-s)^{2}-\epsilon_{3}
$$

and we can parameterize the NE in terms of the three parameters $\epsilon_{1}=e-c, \epsilon_{2}=a+b+d+e$ and $\epsilon_{3}=a+2 b-c+2 e$. Substituting $v_{1}, v_{2}, v_{3}$ into Eq. (17) we find

$$
\begin{aligned}
& \Pi_{A}\left(x^{*}, y^{*}\right)-\Pi_{A}\left(x, y^{*}\right) \\
= & \left(x^{*}-x\right)\left[y^{*} \Delta_{3} v_{3}+\Delta_{1} v_{1}-\Delta_{2} v_{2}\right] \\
= & \left(x^{*}-x\right)(r-s)\left[\Delta_{3}\left\{y^{*}\left(r+\frac{\epsilon_{1}-\epsilon_{3}}{r-s}\right)+\left(1-y^{*}\right)\left(s+\frac{\epsilon_{1}}{r-s}\right)\right\}-\Delta_{2}\left(1+\frac{\epsilon_{1}-\epsilon_{2}}{r-s}\right)\right] .
\end{aligned}
$$

The corresponding inequality for Bob is then obtained by interchanging $x$ and $y$. This gives us the general conditions for a NE in the non-factorizable case. Note that if we set $\epsilon_{1}=\epsilon_{2}=\epsilon_{3}=0$ we recover our previous results in Eq. (20) and Eq. (21). It is also easily 
shown that this condition also implies that $a=b=c=d=e=0$ and so we will recover the factorizable payoff relation shown in Eq. (22)

\section{A. Non-factorizable game with classical embedding}

The embedding of the classical game is obtained by taking $r=1$ and $s=0$, which from Eq. (35) gives the equation for NE

$$
\begin{aligned}
& \Pi_{A}\left(x^{*}, y^{*}\right)-\Pi_{A}\left(x, y^{*}\right) \\
= & \left(x^{*}-x\right)\left[\Delta_{3}\left\{y^{*}\left(1-\epsilon_{3}\right)+\epsilon_{1}\right\}-\Delta_{2}\left(1+\epsilon_{1}-\epsilon_{2}\right)\right] \\
& \Pi_{B}\left(x^{*}, y^{*}\right)-\Pi_{B}\left(x^{*}, y\right) \\
= & \left(y^{*}-y\right)\left[\Delta_{3}\left\{x^{*}\left(1-\epsilon_{3}\right)+\epsilon_{1}\right\}-\Delta_{2}\left(1+\epsilon_{1}-\epsilon_{2}\right)\right] .
\end{aligned}
$$

Thus we have obtained the general conditions for the NE for a non-factorizable table of probabilities, which will embed the classical game when it becomes factorizable. The payoff given by

$$
\begin{aligned}
\Pi_{A}(x, y) & =a_{4}+c\left(a_{1}-a_{4}\right)-d\left(2 a_{4}-a_{2}-a_{3}\right)+x y \Delta_{3}\left(1-\epsilon_{3}\right) \\
& +x\left[\left(a_{2}-a_{4}\right)\left(1-\epsilon_{2}\right)+\epsilon_{1}\left(a_{1}-a_{3}\right)\right]+y\left[\left(a_{3}-a_{4}\right)\left(1-\epsilon_{2}\right)+\epsilon_{1}\left(a_{1}-a_{2}\right)\right]
\end{aligned}
$$

and similarly for Bob.

These produce Table VII and we see that we must have $a, b, c, d, e \geq 0$.

Bob

\begin{tabular}{c}
\multicolumn{2}{c|}{$\begin{array}{c}S_{1}^{\prime} \\
\end{array}$} \\
\end{tabular}

TABLE VII: Non-factorizable probabilities that embed the classical game. 


\section{B. The CHSH inequalities}

Cereceda [43] finds the CHSH sum of correlations [4] for any set of local hidden variables satisfying the causal communication constraint as

$$
\Delta=2\left(p_{1}+p_{4}+p_{5}+p_{8}+p_{9}+p_{12}+p_{14}+p_{15}-2\right)
$$

From Table VII, we can find

$$
\Delta=4(a-c+2 e-1 / 2) .
$$

Inspecting the table of probabilities, we note that $a-c+2 e-\frac{1}{2} \in[-1,1]$, therefore, a range of possible $\Delta \in[-4,4]$ exist in agreement with the expected range [43]. For example, using $a=\frac{1}{2}, b=0, c=0, d=\frac{1}{2}, e=\frac{1}{2}$, we find $\Delta=4$. However, quantum mechanics enforces extra restrictions on the joint probabilities considered here that can arise, namely Cirel'son's bound [44] of $\Delta \in[-2 \sqrt{2}, 2 \sqrt{2}]$. That is, for a physically realizable quantum game, we will have extra restriction on the table of probabilities

$$
|a-c+2 e-1 / 2| \leq 1 / \sqrt{2} .
$$

\section{Quantum Prisoners' Dilemma constructed from non-factorizable joint proba-}

\section{bilities}

For PD, we usually take [8] $a_{1}=3, a_{2}=0, a_{3}=5$, and $a_{4}=1$ in matrices (2) and the strategy pair $\left(x^{*}, y^{*}\right)=(0,0)$ is a NE at which both players receive the payoffs of 1 . To find if non-factorizability permits achieving $\left(x^{*}, y^{*}\right)=(1,1)$ as a NE we note from Eq. (45) that this requires

$$
\Delta_{3}\left(1-\epsilon_{3}+\epsilon_{1}\right)-\Delta_{2}\left(1+\epsilon_{1}-\epsilon_{2}\right) \geq 0 .
$$

Here we refer to a result in Ref. [43] giving a set of non-factorizable joint probabilities that saturates Cirel'son's bound, while maximally violating CHSH inequality. For this set we have $a=d=e=\frac{1}{8}(2+\sqrt{2})$ and $b=c=\frac{1}{2}-\frac{1}{8}(2+\sqrt{2})$. This results in $1-\epsilon_{3}+\epsilon_{1}=0$ and $1+\epsilon_{1}-\epsilon_{2}=0$, and we have the situation of non-factorizability giving $\left(x^{*}, y^{*}\right)=(1,1)$ as a NE. From Eq. (37) we find that the payoff for each player at this NE as

$$
\Pi_{A}(1,1)=\Pi_{B}(1,1)=\frac{1}{8}(18+\sqrt{2})=2.42678,
$$

which is above the payoff of 1 to each player at the classical $\mathrm{NE}$ of $\left(x^{*}, y^{*}\right)=(0,0)$ and is close to the Pareto optimum payoff of 3 for each player. 


\section{Stag Hunt game with non-factorizable joint probabilities}

From Eq. (36), we now have the mixed NE given by

$$
y^{*}=\frac{\Delta_{2}\left(1+\epsilon_{1}-\epsilon_{2}\right)}{\Delta_{3}}-\frac{\epsilon_{1}}{1-\epsilon_{3}},
$$

where we find $\left(1+\epsilon_{1}-\epsilon_{2}\right) \in[-1,1], \epsilon_{1} \in\left[-1, \frac{1}{2}\right]$ and $1-\epsilon_{3} \in\left[-\frac{1}{2}, 1\right]$, so that any mixed NE we desire in the range [0,1], as well as returning to the classical NE when $\epsilon_{1}=\epsilon_{2}=\epsilon_{3}=0$. If we desire to produce the non-classical NE of $\left(x^{*}, y^{*}\right)=(0,1)$ and $\left(x^{*}, y^{*}\right)=(1,0)$, then from Eq. (36) we have the conditions

$$
\begin{aligned}
& \Pi_{A}(1,0)-\Pi_{A}(x, 0)=(1-x)\left(\Delta_{3} \epsilon_{1}-\Delta_{2}\left(1+\epsilon_{1}-\epsilon_{2}\right)\right) \geq 0 \\
& \Pi_{B}(0,1)-\Pi_{B}(0, y)=(1-y)\left(\Delta_{3} \epsilon_{1}-\Delta_{2}\left(1+\epsilon_{1}-\epsilon_{2}\right)\right) \geq 0 .
\end{aligned}
$$

We have $\Delta_{3}>\Delta_{2}>0$ for the SH game and so we can see that if we select $\epsilon_{1} \geq 1+\epsilon_{1}-\epsilon_{2}$,

then we will have achieved this new NE. This condition gives $\epsilon_{2} \geq 1$ or $a+b+d+e \geq 1$. This is easily satisfied, with $b=d=1 / 2$ with the other terms zero, for example.

\section{E. Chicken game with non-factorizable joint probabilities}

The Chicken game is defined with $\Delta_{2}, \Delta_{3}<0$ whereas the SH game has $\Delta_{2}, \Delta_{3}>0$. Thus we can carry over the results from the previous section, except that the NE will invert due to the extra minus sign in Eq. (45).

\section{DISCUSSION}

Quantum versions of $2 \times 2$ games are developed considering the peculiarities of a set of quantum mechanical joint probabilities. The probability sets we consider consist of normalized probabilities satisfying causal communication and the symmetry constraints. Players are allowed classical strategies only and their payoff relations are re-expressed in terms of the joint probabilities. We allow a quantum game thus defined to reduce itself to the classical mixed-strategy game when the set of joint probabilities can be factorized in terms of the factorization parameters $r$ and $s$. Constraints on the parameters $r$ and $s$ are obtained with which this reduction can be realized. 
Non-factorizable sets of joint probabilities are introduced and appropriate parameters $\epsilon_{1}, \epsilon_{2}$, and $\epsilon_{3}$ describing non-factorizability are identified. Quantum games are now constructed by retaining the obtained constraints on the parameters $r$ and $s$ and allowing non-factorizability parameters $\epsilon_{1}, \epsilon_{2}$, and $\epsilon_{3}$ to take non-zero values. Two types of games are identified: Firstly, with $\epsilon_{1}, \epsilon_{2}, \epsilon_{3}=0$, and $r=1, s=0$, we obtain the original classical mixed-strategy game along with its bilinear payoff structure. Secondly, while enforcing $r=1$ and $s=0$, but allowing non-factorizability parameters $\epsilon_{1}, \epsilon_{2}, \epsilon_{3}$ to take non-zero values, we obtain an extension of the classical mixed-strategy game in which the full original classical game, along with its bilinear payoff structure, remains embedded when $\epsilon_{1}, \epsilon_{2}, \epsilon_{3}=0$. We investigate PD within this setup to find that when Cirel'son's bound is maximally saturated, a non-factorizable and quantum game gives the $\mathrm{NE}$ of $\left(x^{*}, y^{*}\right)=(1,1)$ at which both players' payoffs approach to their Pareto optimum value. For the SH we observe that two new and non-classical NE of $\left(x^{*}, y^{*}\right)=(0,1),(1,0)$ can be realized with non-factorizable joint probabilities. We demonstrate that the our non-factorizable extension of the classical game permits us to study situations that are not even physically realizable. That is, the situations in which the corresponding CHSH inequality is violated beyond Cirel'son's bound. We then obtain a constraint, given by Eq. (41), that defines the boundaries of what quantum mechanics can permit for the extension of the original classical $2 \times 2$ game.

Notice that the new parameters $a, b, c, d$ and $e$ are introduced in order to give an idea of the extent of how much non-factorizable a given table of joint probabilities is relative to the factorizable situation. So as to obtain compact expression, the parameters $\epsilon_{1}, \epsilon_{2}, \epsilon_{3}$ are then introduced, each of which depends on $a, b, c, d$ and $e$. These extra parameters are added numerically to the $r$ and $s$ parameters without affecting the meaning of the $r$ and $s$ parameters. It turns out that $r$ and $s$ subsequently become redundant in our quantum game as we set $r=1$ and $s=0$ in order to embed the classical game within the quantum game.

The extension of game theory based on the considerations of quantum mechanical joint probabilities attaining the peculiar character of being non-factorizable has already been investigated in earlier publications [28, 31, 33, 35]. The present manuscript's contribution consists in understanding how placing an extra symmetry requirement on joint probabilities changes the role of non-factorizability in the construction of quantum games. Using probabilistic considerations only, this work explores quantum games that are constructed using an EPR type setting. This approach gives a more accessible perspective on the nature of quan- 
tum mechanical joint probabilities and their potential exploitation in giving an extension to game theory.

One important benefit of this extension is the extended perspective it provides of looking at the quantum mechanical probabilities that is able to cover the classical factorizable, nonfactorizable, and even those situations that quantum mechanics does not allow, within a single framework. Quantum mechanics is a probabilistic theory and this paper holds that probabilistic considerations permit us to have a more clear vision and sense of what quantum mechanics can achieve and what are its limits. From this viewpoint we give an extension to game theory, while focussing on purely probabilistic considerations. We observe that this extension is general enough to show us the classical factorizable situations as well as the situations that are beyond quantum mechanics.

We are motivated to have an entirely probabilistic approach towards quantum games that encompasses classical, quantum and also those hypothetical situations that cannot be realized quantum mechanically. We allow players the same sets of classical strategies so that this scheme is not subjected to Enk and Pike type argumentation. As an EPR type apparatus is used in the playing of the two-player quantum games, their physical realization will involve performing EPR type experiments. These experiments are agreed to entail genuinely quantum features. The sets of joint probabilities, whose non-factorizable property we use in constructing our quantum games, are relevant to generalized EPR type experiments.

The potential benefits of this approach consists of developing an entirely probabilistic understanding of multi-party strategic situations. In these situations, quantum probabilities become crucial in achieving one or the other outcome. The extension of game theory advocated in this paper, rather than being imaginary, is simply a more generic framework, that allows us to consider, in entirely probabilistic terms, also that peculiar domain which resides beyond the bounds of quantum mechanics. We find that, within our probabilistic approach toward quantum games, this domain becomes more easily identifiable, along with easily recognizable classical factorizable and the quantum mechanical non-factorizable domains.

[1] K. Binmore, Game Theory: A Very Short Introduction (Oxford University Press, USA, 2007). 
[2] E. Rasmusen, Games $\mathcal{E}$ Information: An Introduction to Game Theory (Blackwell Publishers Ltd., Oxford, 2001) 3rd Edition.

[3] M. A. Nielsen and I. L. Chuang, Quantum Computation and Quantum Information, Cambridge University Press (2000).

[4] A. Peres, Quantum Theory: Concepts and Methods (Kluwer Academic Publishers, 1995).

[5] N.D. Mermin, Am. J. Phys. 58 (1990) 731.

[6] N.D. Mermin, Phys. Rev. Lett. 65 (1990) 1838.

[7] D.A. Meyer, Phys. Rev. Lett. 82 (1999) 1052.

[8] J. Eisert, M. Wilkens, and M. Lewenstein, Phy. Rev. Lett. 83 (1999) 3077. See also, J. Eisert and M. Wilkens, J. Mod. Opt. 47 (2000) 2543.

[9] L. Vaidman, Found. Phys. 29 (1999) 615.

[10] H.E. Brandt, Prog. Quant. Elect. 22 (1999) 257.

[11] S.C. Benjamin and P. M. Hayden, Phys. Rev. Lett. 87 (2001) 069801.

[12] S.C. Benjamin and P. M. Hayden, Phys. Rev. A 64 (2001) 030301.

[13] S.J. van Enk, R. Pike, Phys Rev A 66 (2002), 024306.

[14] N.F. Johnson, Phys. Rev. A 63 (2001) 020302.

[15] C.F. Lee and N.F. Johnson, Phys. Rev. A 67 (2003) 022311.

[16] L. Marinatto and T. Weber, Phys. Lett. A 272 (2000) 291.

[17] A. Iqbal and A.H. Toor, Phys. Lett. A 280 (2001) 249.

[18] J. Du, H. Li, X. Xu, X. Zhou, and R. Han, Phys. Lett. A 302 (2002) 229.

[19] J. Du, H. Li, X. Xu, M. Shi, J. Wu, X. Zhou and R. Han, Phys. Rev. Lett. 88 (2002) 137902.

[20] E.W. Piotrowski and J. Sladkowski, Physica A 312 (2002) 208.

[21] E.W. Piotrowski and J. Sladkowski, Int. J. Theor. Phys. 42 (2003) 1089.

[22] A.P. Flitney and D. Abbott, Fluct. Noise Lett. 2 (2002) R175.

[23] A. Iqbal, J. Phys. A: Math. Gen. 37 (2004) L353.

[24] A.P. Flitney and D. Abbott, J. Phys. A 38 (2005) 449.

[25] T. Cheon and I. Tsutsui, Phys. Lett. A 348 (2006) 147.

[26] J. Shimamura, S.K. Özdemir, F. Morikoshi and N. Imoto, Int. J. Quant. Inf. 2 (2004) 79.

[27] T. Ichikawa and I. Tsutsui, Annals of Physics 322 (2007) 531.

[28] A. Iqbal and T. Cheon, Proc. SPIE 6802 (2007) 68020A.

[29] A. Iqbal and S. Weigert, J. Phys. A 37 (2004) 5873. 
[30] S.K. Özdemir, J. Shimamura, and N. Imoto, New J. Phys. 9 (2007) 43.

[31] A. Iqbal and T. Cheon, Phys. Rev. E 76 (2007) 061122.

[32] A.P. Flitney and L.C.L. Hollenberg, Phys. Lett. A 363 (2007) 381.

[33] A. Iqbal, T. Cheon and D. Abbott, Phys. Lett. A 372 (2008) 6564.

[34] T. Ichikawa, I. Tsutsui, and T. Cheon, J. Phys. A, Math. Theor. 41 (2008) 135303.

[35] A. Iqbal and D. Abbott, J. Phys. Soc. Jpn. 78 (2009) 014803.

[36] J.M. Chappell, A. Iqbal, M.A. Lohe, L. von Smekal, J. Phys. Soc. Jpn. 78 (2009) 054801.

[37] C. Schmid, A.P. Flitney, W. Wieczorek, N. Kiesel, H. Weinfurter, and L.C.L. Hollenberg, arXiv:0901.0063

[38] R. Dawkins, The Selfish Gene, (Oxford University Press, Oxford, 1976).

[39] A. Einstein, B. Podolsky and N. Rosen, Phys. Rev. 47 (1935) 777.

[40] D. Bohm, Quantum Theory, (Prentice-Hall, Englewood Cliffs, NJ, 1951), pp. 611-623.

[41] J.S. Bell, Speakable and Unspeakable in Quantum Mechanics (Cambridge University Press, Cambridge, 1987).

[42] A. Aspect, J. Dalibard, and G. Roger, Phys. Rev. Lett. 49 (1982) 1804.

[43] J.L. Cereceda, Found. Phys. Lett. 13 (2000) 427.

[44] B.S. Cirel'son, Lett. Math. Phys. 4 (1980) 93. 